\title{
Evaluating Consulting Firms Using a Centroid Ranking Approach based Fuzzy MCDM Method
}

\author{
Ta-Chung Chu \\ Department of Management and Information Technology \\ Southern Taiwan University, Taiwan, R.O.C. \\ E-mail: tcchu@mail.stut.edu.tw
}

\begin{abstract}
The purpose of this paper is to evaluate and select consulting firms by suggesting a centroid ranking approach based fuzzy multiple criteria decision making (MCDM) method, where ratings of alternatives versus qualitative criteria and the different importance weights among criteria are assessed in linguistic values represented by fuzzy numbers. Criteria are categorized to qualitative, benefit quantitative and cost quantitative ones. Membership functions of the final fuzzy evaluation values can be developed through $\alpha$-cuts and interval arithmetic. The ranking approach of Euclidean distance based on centroid points is applied to determine the ranking order of all alternatives. Formulas for the ranking procedure can be clearly derived. Finally, a numerical example is used to demonstrate the computational process of the suggested model.
\end{abstract}

Keywords: Consulting firms, Fuzzy MCDM, Centroid, Ranking

\section{Introduction}

To survive in tight competition in today's business world, a company usually develops a new product which is different from, or better than, that of its competitors. A crucial factor such as pricing must be determined when introducing a new product to the market because it is very sensitive to customers. Wrong pricing strategy for a new product developed from a heavy investment can lead a company into loss or even bankruptcy. However, determining the best pricing strategy for a new product is difficult and many factors must be considered. To cautiously deal with this problem, many companies usually ask for consulting firm service. Thus evaluating and selecting a suitable consulting firm becomes an important issue.

A consulting firm is a firm of experts providing professional advice to an organization for a fee. A consulting firm consists of consultants who are experts in their field. For some global consulting firms, their employees represent from many nationality. Usually, a consulting firm provides its service which is in core business discipline, from marketing to operations; but there are consulting firms which not only provide business service but politics as well.

Many criteria must be considered when evaluating consulting firms, some of them are qualitative, such as reputation, some are quantitative, such as firm size; moreover, criteria may have different importance. Therefore, how to comprehensively aggregate these criteria and importance weights becomes a critical issue in effectively evaluating consulting firms. Some relevant works have been studied in the evaluation of consulting firms. Creplets, et al. ${ }^{[13]}$ (2001) analyzed theoretically and empirically the differences between consultants and experts in the framework of the knowledge-based economy in order to introduce the central concepts of epistemic community and community of practice. However they did not talk detail about the other criteria that are supposed to be considered by a consulting firm such as the implementation cost and its knowledge. Wang and Chen $^{[21]}$ (2006) presented how human inputs (top management, users, and external consultants) are linked to communication effectiveness and conflict resolution in the ERP consulting process, as well as the effects of these factors on the quality of the system implemented. Their findings indicated that top management support indirectly enhances ERP system quality through its positive effect on conflict resolution in the consulting process. The results also showed that high user support enhances communication effectiveness; however, communication effectiveness does not influence conflict resolution and ERP system quality. This paper lacks formulas for completing decision making. Altman ${ }^{[3]}$ (2008) indicated what should company consider and give guidelines in choosing the right consultancy. This article gives clear understanding about what is a consulting firm, but is short of quantitative process in aggregating the needed criteria. In 2009, Saremi, et al. ${ }^{[19]}$ used Nominal Group Technique (NGT) in deciding criteria for selecting the best consultant firm. They helped company to choose the best consultant for TQM selection under fuzzy TOPSIS, where qualitative and quantitative criteria are considered. But all criteria, even implemented cost, are regarded as qualitative one. Cebeci and Ruan ${ }^{[7]}$ (2007) provided an analytical tool to select the best quality consultant providing the most customer satisfaction, where fuzzy analytic hierarchy process was applied to compare these consultancy firms. But, all the attributes are qualitative.

To resolve the above problems in evaluating consulting firms, this paper suggests a centroid ranking approach based fuzzy MCDM method, where both subjective (or qualitative) and objective (or quantitative) criteria are considered. In the proposed model, ratings of alternatives versus qualitative criteria and the importance weights of all the criteria are assessed in 
linguistic values represented by fuzzy numbers. Many methods have been proposed to solve fuzzy MCDM problems. A review and comparison of many of these methods can be found in Chen and Hwang ${ }^{[9]}$ (1992), Carlsson and Fullèr ${ }^{[6]}$ (1996), Ribeiro ${ }^{[18]}$ (1996) and Triantaphyllou and $\operatorname{Lin}^{[20]}$ (1996). Some recent applications can be found in $\mathrm{Chen}^{[8]}$ (2001), Chou ${ }^{[10]}$ (2007), Chou et al. ${ }^{[11]}$ (2006) and Önüt et al. ${ }^{[17]}$ (2009). In most fuzzy MCDM problems, the final evaluation values of alternatives are still fuzzy numbers and these fuzzy numbers need a proper ranking approach to defuzzify them into crisp values for decision making. Many approaches for ranking fuzzy numbers have been studied. A review of many of these approaches can be found in Bortolan and Degani ${ }^{[5]}(1985)$, Chen and Huang $^{[9]}$ (1992), and Wang and Kerre ${ }^{[22]}$ (2001). Some recent methods can be seen in Abbasbandy and Asady ${ }^{[1]}$ (2006), Abbasbandy and Hajjari ${ }^{[2]}$ (2009), Asady ${ }^{[4]}$ (2010), Liu and Han ${ }^{[16]}$ (2005), Wang and Lee ${ }^{[23]}$ (2008) and Yong et al. ${ }^{[25]}$ (2006). In spite of advantages, some of these methods are computational complex and difficult to implement and none of them can satisfactorily rank fuzzy numbers in all cases. Moreover, the above works do not provide connection by formulae between the final evaluation values and the ranking procedure, limiting their applicability and computation efficiency.

To resolve these problems, this paper suggests applying the ranking approach of centroid from Wang et al. $^{[24]}$ (2006) to obtain the Euclidean distance to defuzzify all the final fuzzy evaluation values in order to complete the model. Formulae can be clearly developed to present the ranking procedure. Finally, a numerical example demonstrates the computational process of the suggested model.

\section{Fuzzy set theory}

Fuzzy set theory was first introduced by $\operatorname{Zadeh}^{[27]}$ in 1965. It is to deal with problems involving fuzzy phenomena. Also, it has been considered as a useful tool of modeling language represented by fuzzy numbers to approximate system in which fuzzy phenomena exists. Some basic concepts in fuzzy set theory are briefly introduced in the following sections, which can also be seen in the author's previous work ${ }^{[12]}$.

\subsection{Fuzzy set}

The fuzzy set $A$ can be expressed as ${ }^{[15]}$ :

$A=\left\{\left(x, f_{A}(x)\right) \mid x \in U\right\}$

where $U$ is the universe of discourse, $x$ is an element in $U, A$ is a fuzzy set in $U, f_{A}(x)$ is the membership function of $A$ at $x$. The larger $f_{A}(x)$, the stronger the grade of membership for $x$ in $A$.

\subsection{Fuzzy numbers}

A real fuzzy number $A$ is described as any fuzzy subset of the real line $R$ with membership function $f_{A}$ which possesses the following properties ${ }^{[14]}$ :

(a) $f_{A}$ is a continuous mapping from $R$ to $[0,1]$;

(b) $f_{A}(x)=0, \forall x \in(-\infty, a]$;

(c) $f_{A}$ is strictly increasing on $[a, b]$;

(d) $f_{A}(x)=1, x \in[b, c]$;

(e) $f_{A}$ is strictly decreasing on $[c, d]$;

(f) $f_{A}(x)=0, \forall x \in[d, \infty)$;

where $a \leq b \leq c \leq d, A$ can be denoted as $[a, b, c, d]$. The membership function $f_{A}$ of the fuzzy number $A$ can also be expressed as:

$f_{A}(x)= \begin{cases}f_{A}^{L}(x), & a \leq x \leq b \\ 1, & b \leq x \leq c \\ f_{A}^{R}(x), & c \leq x \leq d \\ 0, & \text { otherwise }\end{cases}$

where $f_{A}^{L}(x)$ and $f_{A}^{R}(x)$ are left and right membership functions of $A$, respectively.

\section{3. $\alpha$-cut}

The $\alpha$-cuts of fuzzy number $A$ can be defined as ${ }^{[15]}$.

$A^{\alpha}=\left\{x \mid f_{A}(x) \geq \alpha\right\}, \alpha \in[0,1]$

where $A^{\alpha}$ is a non-empty bounded closed interval contained in $R$ and can be denoted by $A^{\alpha}=\left\lfloor A_{l}^{\alpha}, A_{u}^{\alpha}\right\rfloor$, where $A_{l}^{\alpha}$ and $A_{u}^{\alpha}$ are its lower and upper bounds, respectively.

\subsection{Fuzzy arithmetic operations}

Given fuzzy numbers $A$ and $B, A, B \in R^{+}$, the $\alpha$-cuts of $A$ and $B$ are $A^{\alpha}=\left\lfloor A_{l}^{\alpha}, A_{u}^{\alpha}\right\rfloor$ and $B^{\alpha}=\left\lfloor B_{l}^{\alpha}, B_{u}^{\alpha}\right\rfloor$, respectively. By interval arithmetic, some main operations of $A$ and $B$ can be expressed as follows ${ }^{[15]}$ :

$(A \oplus B)^{\alpha}=\left[A_{l}^{\alpha}+B_{l}^{\alpha}, A_{u}^{\alpha}+B_{u}^{\alpha}\right]$
$(A \ominus B)^{\alpha}=\left[A_{l}^{\alpha}-B_{u}^{\alpha}, A_{u}^{\alpha}-B_{l}^{\alpha}\right]$
$(A \otimes B)^{\alpha}=\left[A_{l}^{\alpha} \cdot B_{l}^{\alpha}, A_{u}^{\alpha} \cdot B_{u}^{\alpha}\right]$
$(A \oslash B)^{\alpha}=\left[A_{l}^{\alpha} / B_{u}^{\alpha}, A_{u}^{\alpha} / B_{l}^{\alpha}\right]$
$(A \otimes r)^{\alpha}=\left[A_{l}^{\alpha} \cdot r, A_{u}^{\alpha} \cdot r\right], r \in R^{+}$

\subsection{Linguistic values}

A linguistic variable is a variable whose values are expressed in linguistic terms. Linguistic variable is a very helpful concept for dealing with situations which are too complex or not well-defined to be reasonably described by traditional quantitative expressions ${ }^{[26]}$. For example, "importance" is a linguistic variable whose values include UI (unimportant), LI (less important), IM 
(important), MI (more important) and VI (very important). These linguistic values can be further represented by triangular fuzzy numbers such as $\mathrm{VL}=(0.0,0.0,0.25), \mathrm{L}=(0.0,0.25,0.5), \mathrm{M}=(0.25,0.5,0.75)$, $\mathrm{H}=(0.5,0.75,1.0)$ and $\mathrm{VH}=(0.75,1.0,1.0)$.

\section{Model establishment}

Assume that a committee of $k$ decision-makers $\left(D_{t}\right.$, $t=1 \sim k$ ) is responsible for evaluating $m$ alternatives ( $A_{i}$, $i=1 \sim m$ ) under $n$ criteria, and criteria are categorized to subjective $\left(C_{j}, j=1 \sim h\right)$ and objective $\left(C_{j}, j=h+1 \sim n\right)$, objective/quantitative criteria are further classified into benefit (B) and cost (C).

\subsection{Average ratings of alternatives versus subjective criteria}

Let $\quad x_{i j t}=\left(o_{i j t}, p_{i j t}, q_{i j t}\right), \quad x_{i j t} \in R^{+}, i=1,2, \ldots, m, j$ $=1,2, \ldots, h, t=1,2, \ldots, k$.

$x_{i j}=\left(\frac{1}{k}\right) \otimes\left(x_{i j 1} \oplus \ldots \oplus x_{i j t} \oplus x_{i j k}\right)$

where $o_{i j}=\sum_{t=1}^{k} \frac{o_{i j t}}{k}, \quad p_{i j}=\sum_{t=1}^{k} \frac{p_{i j t}}{k}, \quad q_{i j}=\sum_{t=1}^{k} \frac{q_{i j t}}{k}, \quad x_{i j t}$ be the rating assigned to $A_{i}$ by $D_{t}$ for $C_{j} \cdot x_{i j}$ is the averaged rating of $A_{i}$ under $C_{j}$.

\subsection{Normalize performance of alternatives versus objective criteria}

Herein, quantitative values of alternatives versus objective criteria are assumed to be uncertain and can be expressed in triangular fuzzy numbers. These values have different units and must be normalized. The normalization is completed by a suggested approach from Chu and $\operatorname{Lin}^{[12]}$ (2009), which preserves the property where the ranges of normalized triangular fuzzy numbers belong to $[0,1]$. Suppose $r_{i j}=\left(e_{i j}, f_{i j}, g_{i j}\right)$ is the performance of alternative $i$ versus objective criterion $j$, the normalized value can be denoted as:

$$
\begin{aligned}
& x_{i j}=\left(\frac{e_{i j}-e_{j}^{*}}{d_{j}^{*}}, \frac{f_{i j}-e_{j}^{*}}{d_{j}^{*}}, \frac{g_{i j}-e_{j}^{*}}{d_{j}^{*}}\right), x \in B \\
& x_{i j}=\left(\frac{g_{j}^{*}-g_{i j}}{d_{j}^{*}}, \frac{g_{j}^{*}-f_{i j}}{d_{j}^{*}}, \frac{g_{j}^{*}-e_{i j}}{d_{j}^{*}}\right), x \in C
\end{aligned}
$$

where $e_{j}^{*}=\min _{i} e_{i j}, \quad g_{j}^{*}=\max _{i} g_{i j}, \quad d_{j}^{*}=g_{j}^{*}-e_{j}^{*}$, $i=1 \sim m, j=h+1 \sim n$. Benefit (B) criterion has the characteristics of the larger the better; while cost $(\mathrm{C})$ criterion has the characteristics of the smaller the better.

\subsection{Average weights}

Let $w_{j t}=\left(a_{j t}, b_{j t}, c_{j t}\right), \quad w_{j t} \in R^{+}, j=1,2, \ldots, n ; \quad t$ $=1,2, \ldots, k$.

$w_{j}=\left(\frac{1}{k}\right) \otimes\left(w_{j 1} \oplus w_{j 2} \oplus \ldots \oplus w_{j k}\right)$

where $a_{j}=\sum_{t=1}^{k} \frac{a_{j t}}{k}, \quad b_{j}=\sum_{t=1}^{k} \frac{b_{j t}}{k}, \quad c_{j}=\sum_{t=1}^{k} \frac{c_{j t}}{k}, \quad w_{j t}$ be the weight assigned by $D_{t}$ to $C_{j} \cdot w_{j}$ is the averaged weight of $C_{j}$ assessed by $k$ decision-makers.

\subsection{Aggregate the weighted ratings}

The aggregation of weighted ratings, $G_{i}$, for each alternative is implemented by the concept of simple additive weighting as:

$G_{i}=\left(\frac{1}{n}\right) \times\left[R_{i 1} \oplus R_{i 2} \oplus \ldots \oplus R_{i j} \oplus \ldots \oplus R_{i n}\right]$

where $R_{i j}=x_{i j} \otimes w_{j}, i=1,2, \ldots, m j=1,2, \ldots, n$.

Let

$\left(o_{i j}, p_{i j}, q_{i j}\right)=\left\{\begin{array}{l}\left(\frac{e_{i j}-e_{j}^{*}}{d_{j}^{*}}, \frac{f_{i j}-e_{j}^{*}}{d_{j}^{*}}, \frac{g_{i j}-e_{j}^{*}}{d_{j}^{*}}\right), j \in B, \\ \left(\frac{g_{j}^{*}-g_{i j}}{d_{j}^{*}}, \frac{g_{j}^{*}-f_{i j}}{d_{j}^{*}}, \frac{g_{j}^{*}-e_{i j}}{d_{j}^{*}}\right), j \in C\end{array}\right.$

By applying Eqs. (4) and (10), Eq. (9) can be developed via arithmetic operation of fuzzy numbers as:

$$
\begin{aligned}
& G_{i}^{\alpha}=\left(\frac{1}{n}\right) \sum_{j=1}^{n} x_{i j}^{\alpha} \otimes w_{j}^{\alpha} \\
& =\left[\frac{1}{n} \sum_{j=1}^{n}\left(p_{i j}-o_{i j}\right)\left(b_{j}-a_{j}\right) \alpha^{2}+\right. \\
& \frac{1}{n} \sum_{j=1}^{n}\left[o_{i j}\left(b_{j}-a_{j}\right)+a_{j}\left(p_{i j}-o_{i j}\right)\right] \alpha+\frac{1}{n} \sum_{j=1}^{n} o_{i j} a_{j}, \\
& \frac{1}{n} \sum_{j=1}^{n}\left(p_{i j}-q_{i j}\right)\left(b_{j}-c_{j}\right) \alpha^{2}+ \\
& \left.\frac{1}{n} \sum_{j=1}^{n}\left[q_{i j}\left(b_{j}-c_{j}\right)+c_{j}\left(p_{i j}-q_{i j}\right)\right] \alpha+\frac{1}{n} \sum_{j=1}^{n} q_{i j} c_{j}\right]
\end{aligned}
$$

Let

$$
\begin{aligned}
& I_{i 1}=\frac{1}{n} \sum_{j=1}^{n}\left(p_{i j}-o_{i j}\right)\left(b_{j}-a_{j}\right), \\
& J_{i 1}=\frac{1}{n} \sum_{j=1}^{n}\left[o_{i j}\left(b_{j}-a_{j}\right)+a_{j}\left(p_{i j}-o_{i j}\right)\right],
\end{aligned}
$$


$I_{i 2}=\frac{1}{n} \sum_{j=1}^{n}\left(p_{i j}-q_{i j}\right)\left(b_{j}-c_{j}\right)$,

$J_{i 2}=\frac{1}{n} \sum_{j=1}^{n}\left[q_{i j}\left(b_{j}-c_{j}\right)+c_{j}\left(p_{i j}-q_{i j}\right)\right]$,

$Q_{i}=\frac{1}{n} \sum_{j=1}^{n} o_{i j} a_{j}, \quad Y_{i}=\frac{1}{n} \sum_{j=1}^{n} p_{i j} b_{j}, \quad Z_{i}=\frac{1}{n} \sum_{j=1}^{n} q_{i j} c_{j}$

By applying the above assumption to Eqs (11), we have two equations to solve:

$I_{i 1} \alpha^{2}+J_{i 1} \alpha+Q_{i}-x=0$

$I_{i 2} \alpha^{2}+J_{i 2} \alpha+Z_{i}-x=0$

The left and right membership functions of $G_{i}$ can be developed as:

$f_{G_{i}}^{L}(x)=\left\{-J_{i 1}+\left[J_{i 1}^{2}+4 I_{i 1}\left(x-Q_{i}\right)\right]^{1 / 2}\right\} / 2 I_{i 1}, Q_{i} \leq x \leq Y_{i} ;$

$f_{G_{i}}^{R}(x)=\left\{-J_{i 2}-\left[J_{i 2}^{2}+4 I_{i 2}\left(x-Z_{i}\right)\right]^{1 / 2}\right\} / 2 I_{i 2}, Y_{i} \leq x \leq Z_{i}$.

For convenience, Eqs. (14) and (15) of $G_{i}$ can be denoted as Eq. (16) as follows:

$G_{i}=\left(Q_{i}, Y_{i}, Z_{i} ; I_{i 1}, J_{i 1} ; I_{i 2}, J_{i 2}\right)$

The inverse functions of the above two equations, (14) and (15), can be produced as:

$g_{G_{i}}^{L}(y)=I_{i 1} y^{2}+J_{i 1} y+Q_{i}, \quad 0 \leq y \leq 1$

$g_{G_{i}}^{R}(y)=I_{i 2} y^{2}+J_{i 2} y+Z_{i}, 0 \leq y \leq 1$

\subsection{Obtain ranking}

The centroid ranking method from Wang et al. ${ }^{[24]}$ (2006) is applied to rank the alternatives and detailed interpretation of centroid equations can be seen in Wang et al.' paper. The centroid on $\mathrm{x}$-axis and $\mathrm{y}$-axis can be shown as follows:

$$
\begin{gathered}
x\left(G_{i}\right)=\frac{\int_{-\infty}^{+\infty} x f_{G_{i}}(x) d x}{\int_{-\infty}^{+\infty} f_{G_{i}}(x) d x} \\
=\frac{\int_{Q_{i}}^{Y_{i}} x f_{G_{i}}^{L}(x) d x+\int_{Y_{i}}^{z_{i}} x f_{G_{i}}^{L}(x) d x}{\int_{Q_{i}}^{Y_{i}} f_{G_{i}}^{L}(x) d x+\int_{Y_{i}}^{z_{i}} f_{G_{i}}^{L}(x) d x}
\end{gathered}
$$

$$
y\left(G_{i}\right)=\frac{\int_{0}^{1} y\left[g_{G_{i}}^{R}(y)-g_{G_{i}}^{L}(y)\right] d y}{\int_{0}^{1}\left[g_{G_{i}}^{R}(y)-g_{G_{i}}^{L}(y)\right] d y}
$$

Then we calculate the Euclidean distances from their centroid points to the origin to defuzzify fuzzy numbers. The larger the distance is, the higher priority fuzzy number is ranked.

$\sqrt{x^{2}\left(G_{i}\right)+y^{2}\left(G_{i}\right)}$

Formulas for centroids on $\mathrm{x}$-axis and $\mathrm{y}$-axis can be developed as follows:

$$
\begin{aligned}
& \int_{Q_{i}}^{Y_{i}} x f_{G_{i}}^{L}(x) d x \\
& =\int_{Q_{i}}^{Y_{i}} x\left[\left\{-J_{i 1}+\left[J_{i 1}^{2}+4 I_{i 1}\left(x-Q_{i}\right)\right]^{1 / 2}\right\} / 2 I_{i 1}\right] d x \\
& =\frac{J_{i 1}\left(Q_{i}^{2}-Y_{i}^{2}\right)}{4 I_{i 1}}+\frac{1}{2 I_{i 1}} \int_{Q_{i}}^{Y_{i}}\left[J_{i 1}^{2}+4 I_{i 1}\left(x-Q_{i}\right)\right]^{1 / 2} x d x
\end{aligned}
$$

Let $\left[J_{i 1}^{2}+4 I_{i 1}\left(x-Q_{i}\right)\right]^{1 / 2}=t$

$$
\begin{aligned}
& x=\frac{t^{2}+4 I_{i 1} Q_{i}-J_{i 1}^{2}}{4 I_{i 1}}, \quad d x=\frac{t d t}{2 I_{i 1}} \\
& x=Q_{i} \Rightarrow t=\left(4 Q_{i} I_{i 1}+J_{i 1}^{2}-4 I_{i 1} Q_{i}\right)^{1 / 2}=J_{i 1} \\
& x=Y_{i} \Rightarrow t=\left(4 Y_{i} I_{i 1}+J_{i 1}^{2}-4 I_{i 1} Q_{i}\right)^{1 / 2}
\end{aligned}
$$

Assume $\left(4 Y_{i} I_{i 1}+J_{i 1}^{2}-4 I_{i 1} Q_{i}\right)^{1 / 2}=Y_{i}^{\prime}$, we obtain:

$$
\begin{aligned}
& \frac{1}{2 I_{i 1}} \int_{Q_{i}}^{Y_{i}}\left[J_{i 1}^{2}+4 I_{i 1}\left(x-Q_{i}\right)\right]^{1 / 2} x d x \\
& =\frac{1}{2 I_{i 1}} \int_{J_{i 1}}^{Y_{i}^{\prime}} \frac{t^{2}+4 I_{i 1} Q_{i}-J_{i 1}^{2} t}{4 I_{i 1}} \frac{t d t}{2 I_{i 1}} \\
& =\frac{1}{16 I_{i 1}^{3}}\left[\frac{Y_{i}^{\prime 5}-J_{i 1}^{5}}{5}+\left(4 I_{i 1} Q_{i}-J_{i 1}^{2}\right) \frac{Y_{i}^{\prime 3}-J_{i 1}^{3}}{3}\right] \\
& \int_{Q_{i}}^{Y_{i}} x\left[\left\{-J_{i 1}+\left[J_{i 1}^{2}+4 I_{i 1}\left(x-Q_{i}\right)\right]^{1 / 2}\right\} / 2 I_{i 1}\right] d x \\
& =\frac{J_{i 1}\left(Q_{i}^{2}-Y_{i}^{2}\right)}{4 I_{i 1}}+\frac{1}{16 I_{i 1}^{3}}\left[\frac{Y_{i}^{\prime 5}-J_{i 1}^{5}}{5}+\left(4 I_{i 1} Q_{i}-J_{i 1}^{2}\right) \frac{Y_{i}^{\prime 3}-J_{i 1}^{3}}{3}\right]
\end{aligned}
$$

Similarly,

$$
\begin{aligned}
& \int_{Y_{i}}^{Z_{i}} x f_{G_{i}}^{R}(x) d x \\
& =\int_{Y_{i}}^{Z_{i}} x\left[\left\{-J_{i 2}+\left[J_{i 2}^{2}+4 I_{i 2}\left(x-Z_{i}\right)\right]^{1 / 2}\right\} / 2 I_{i 2}\right] d x
\end{aligned}
$$




$$
\begin{aligned}
& =\frac{J_{i 2}\left(Y_{i}^{2}-Z_{i}^{2}\right)}{4 I_{i 2}}-\frac{1}{16 I_{i 2}^{3}}\left[\frac{J_{i 2}^{5}-Y_{i}^{\prime \prime}}{5}+\left(4 I_{i 2} Z_{i}-J_{i 2}^{2}\right) \frac{J_{i 2}^{3}-Y_{i}^{\prime \prime}}{3}\right] \\
& \int_{Q_{i}}^{Y_{i}} f_{G_{i}}^{L}(x) d x \\
& =\int_{Q_{i}}^{Y_{i}}\left[\left\{-J_{i 1}+\left[J_{i 1}^{2}+4 I_{i 1}\left(x-Q_{i}\right)\right]^{1 / 2}\right\} / 2 I_{i 1}\right] d x \\
& =\frac{J_{i 1}\left(Q_{i}-Y_{i}\right)}{2 I_{i 1}}+\frac{Y_{i}^{3}-J_{i 1}^{3}}{12 I_{i 1}^{2}} \\
& \int_{Y_{i}}^{Z_{i}} f_{G_{i}}^{R}(x) d x \\
& =\int_{Y_{i}}^{Z_{i}}\left[\left\{-J_{i 2}+\left[J_{i 2}^{2}+4 I_{i 2}\left(x-Z_{i}\right)\right]^{1 / 2}\right\} / 2 I_{i 2}\right] d x \\
& =\frac{J_{i 2}\left(Y_{i}-Z_{i}\right)}{2 I_{i 2}}+\frac{J_{i 2}^{3}-Y_{i}^{\prime \prime}}{12 I_{i 2}^{2}}
\end{aligned}
$$

By applying Eqs. (22)-(27), Eq. (19) can be produced as:

$$
\frac{\frac{x\left(G_{i}\right)=}{4 I_{i 1}}\left(Q_{i}^{2}-Y_{i}^{2}\right)}{4 I_{i 1}}+\frac{1}{16 l_{i 1}^{3}}\left[\frac{Y_{i}^{15}-J_{i 1}^{5}}{5}+\left(4 I_{i 1} Q_{i}-J_{i 1}^{2}\right) \frac{\left.Y_{i}^{3}-\frac{J_{i 1}^{3}}{3}\right]+\frac{J_{i 2}\left(Y_{i}^{2}-Z_{i}^{2}\right)}{4 I_{i 2}}-\frac{1}{16 I_{i 2}^{3}}\left[\frac{J_{i 2}^{5}-Y_{i}^{15}}{5}+\left(4 I_{i 2} Z_{i}-J_{i 2}^{2}\right) \frac{J_{i 2}^{3}-Y_{i}^{13}}{3}\right]}{\frac{J_{i 1}\left(Q_{i}-Y_{i}\right)}{2 I_{i 1}}+\frac{Y_{i}^{3}-J_{i 1}^{3}}{12 I_{i 1}^{2}}+\frac{J_{i 2}\left(Y_{i}-Z_{i}\right)}{2 I_{i 2}}-\frac{J_{i 2}^{3}-Y_{i}^{13}}{122_{i 2}^{2}}}\right.
$$

Similarly, Eq. (20) can be expressed as:

$$
\begin{aligned}
& y\left(G_{i}\right) \\
= & \frac{\int_{0}^{1} y\left[\left(I_{i 2} y^{2}+J_{i 2} y+Z_{i}\right)-\left(I_{i 1} y^{2}+J_{i 1} y+Q_{i}\right)\right] d y}{\int_{0}^{1}\left[\left(I_{i 2} y^{2}+J_{i 2} y+Z_{i}\right)-\left(I_{i 1} y^{2}+J_{i 1} y+Q_{i}\right)\right] d y} \\
= & \frac{\frac{I_{i 2}-I_{i 1}}{4}+\frac{J_{i 2}-J_{i 1}}{3}+\frac{Z_{i}-Q_{i}}{2}}{\frac{I_{i 2}-I_{i 1}}{3}+\frac{J_{i 2}-J_{i 1}}{2}+Z_{i}-Q_{i}}
\end{aligned}
$$

Then, Eqs. (28)-(29) are applied to Eq. (21) to produce the Euclidean distances of each fuzzy number. Finally we can rank the priority of segment alternatives based on values of Euclidean distances. Company is suggested to choose alternative candidate which has highest priority as its consulting firm.

\section{Numerical example}

This section uses an artificial numerical example to demonstrate the computational process of the suggested model. Suppose a manufacturing company must select a consulting firm to help determine the price for its new product. After preliminary screening, four alternative consulting firms, $A_{1}, A_{2}, A_{3}$ and $A_{4}$, are chosen for further evaluation. A committee of five decision-makers $D_{1}, D_{2}, D_{3}, D_{4}$ and $D_{5}$ is formed to conduct the evaluation and selection of the four alternative consulting firms. Further suppose three benefit qualitative criteria, such as reputation $\left(C_{1}\right)$, technical skills $\left(C_{2}\right)$, and knowledge of business $\left(C_{3}\right)$, as well as three benefit quantitative criteria, such as company size $\left(C_{4}\right.$, \# of employees, unit: hundred $)$, potential profit $\left(C_{5}\right.$, unit: million \$) and expected growth $\left(C_{6}\right.$, unit: $\left.\%\right)$, and one cost quantitative criterion, such as $\operatorname{cost}\left(C_{7}\right.$, unit: 10 thousand \$), are considered.

Moreover, suppose that the decision-makers use the linguistic rating set a linguistic rating set $W=\{\mathrm{VL}, \mathrm{L}, \mathrm{M}$, $\mathrm{H}, \mathrm{VH}\}$, where $\mathrm{VL}=$ Very $\mathrm{Low}=(0,0.1,0.3)$, $\mathrm{L}=\mathrm{Low}=(0.1,0.3,0.5), \quad \mathrm{M}=$ Medium $=(0.3,0.5,0.7)$, $\mathrm{H}=\mathrm{High}=(0.5,0.7,0.9)$, and $\mathrm{VH}=$ Very $\mathrm{High}=(0.7,0.9,1)$, to evaluate the suitability of consulting firms under each of the qualitative criteria. In addition, the decision-makers employ a linguistic weighting set $W=\{\mathrm{UI}, \mathrm{LI}, \mathrm{IM}, \mathrm{MI}, \mathrm{VI}\}$ where $\mathrm{UI}=$ Unimportant $=(0.0,0.0,0.25), \quad$ LI=Less Important $=(0.0,0.25,0.5)$,

$\mathrm{IM}=$ Important $=(0.25,0.5,0.75), \quad \mathrm{MI}=$ More Important $=(0.5,0.75,1.0), \quad$ and $\quad \mathrm{VI}=$ Very Important $=(0.75,1.0,1.0)$, to assess the importance of all the criteria. Moreover, suppose that the suitability ratings of consulting firms versus the five qualitative criteria from the three decision-makers are presented in Table 1, values of consulting firms under quantitative criteria are presented in Table 3, and the importance weights of all criteria are presented in Table 6 .

By Eq. (5), the averaged suitability ratings of consulting firm $A_{i}$ under each qualitative criterion $C_{j}$ from the decision-making committee can be obtained as shown in Table 2. By Eqs. (6)-(7), normalized values of consulting firm $A_{i}$ versus each quantitative criterion can be obtained and are displayed in Tables 4-5. Through Eq. (8), the averaged weights of criteria from the decision-making committee can be obtained as presented in Table 7.

Through Eqs. (9)-(16), the membership functions of the final fuzzy evaluation values, $G_{i}, i=1 \sim 4$, can be produced in Table 8. Through Eqs. (17)-(29), centroid points can be produced as shown in Table 9 and the Euclidean distances for the alternative market segments can then be obtained as shown in Table 10 .

Table 1. Ratings of consulting firms versus qualitative criteria

\begin{tabular}{ccccccc}
\hline A. & $C$. & $D_{1}$ & $D_{2}$ & $D_{3}$ & $D_{4}$ & $D_{5}$ \\
\hline \multirow{4}{*}{$A_{1}$} & $C_{1}$ & $\mathrm{H}$ & $\mathrm{M}$ & $\mathrm{H}$ & $\mathrm{H}$ & $\mathrm{VH}$ \\
& $C_{2}$ & $\mathrm{H}$ & $\mathrm{H}$ & $\mathrm{VH}$ & $\mathrm{H}$ & $\mathrm{H}$ \\
& $C_{3}$ & $\mathrm{H}$ & $\mathrm{H}$ & $\mathrm{L}$ & $\mathrm{M}$ & $\mathrm{H}$ \\
& $C_{1}$ & $\mathrm{~L}$ & $\mathrm{M}$ & $\mathrm{VH}$ & $\mathrm{H}$ & $\mathrm{H}$ \\
$A_{2}$ & $C_{2}$ & $\mathrm{VH}$ & $\mathrm{H}$ & $\mathrm{VH}$ & $\mathrm{VH}$ & $\mathrm{H}$ \\
& $C_{3}$ & $\mathrm{~L}$ & $\mathrm{VL}$ & $\mathrm{M}$ & $\mathrm{M}$ & $\mathrm{H}$ \\
& $C_{1}$ & $\mathrm{H}$ & $\mathrm{M}$ & $\mathrm{VL}$ & $\mathrm{M}$ & $\mathrm{M}$ \\
$A_{3}$ & $C_{2}$ & $\mathrm{M}$ & $\mathrm{L}$ & $\mathrm{M}$ & $\mathrm{L}$ & $\mathrm{M}$ \\
& $C_{3}$ & $\mathrm{~L}$ & $\mathrm{H}$ & $\mathrm{L}$ & $\mathrm{VL}$ & $\mathrm{VL}$ \\
& $C_{1}$ & $\mathrm{VH}$ & $\mathrm{L}$ & $\mathrm{VL}$ & $\mathrm{M}$ & $\mathrm{VL}$ \\
$A_{4}$ & $C_{2}$ & $\mathrm{M}$ & $\mathrm{L}$ & $\mathrm{M}$ & $\mathrm{VL}$ & $\mathrm{M}$ \\
& $C_{3}$ & $\mathrm{VL}$ & $\mathrm{L}$ & $\mathrm{VL}$ & $\mathrm{VL}$ & $\mathrm{L}$ \\
\hline
\end{tabular}


Table 2. Averaged ratings of consulting firms versus qualitative criteria

\begin{tabular}{cll}
\hline A. & $C$. & Averaged ratings \\
\hline \multirow{3}{*}{$A_{1}$} & $C_{1}$ & $(0.50,0.70,0.88)$ \\
& $C_{2}$ & $(0.54,0.74,0.92)$ \\
& $C_{3}$ & $(0.38,0.58,0.78)$ \\
$A_{2}$ & $C_{1}$ & $(0.42,0.62,0.80)$ \\
& $C_{2}$ & $(0.62,0.82,0.96)$ \\
& $C_{3}$ & $(0.24,0.42,0.62)$ \\
$A_{3}$ & $C_{1}$ & $(0.28,0.46,0.66)$ \\
& $C_{2}$ & $(0.22,0.42,0.62)$ \\
& $C_{3}$ & $(0.14,0.30,0.50)$ \\
$A_{4}$ & $C_{1}$ & $(0.22,0.38,0.56)$ \\
& $C_{2}$ & $(0.20,0.38,0.58)$ \\
& $C_{3}$ & $(0.04,0.18,0.38)$ \\
\hline
\end{tabular}

Table 3. Numerical values of quantitative criteria

\begin{tabular}{ccccc}
\hline$C$. & $A_{1}$ & $A_{2}$ & $A_{3}$ & $A_{4}$ \\
\hline$C_{4}$ & $(25,30,37.5)$ & $(8,9.3,10)$ & $(36,43.7,55)$ & $(5,7.5,8.97)$ \\
$C_{5}$ & $(2,3,6)$ & $(6.5,8,12)$ & $(2.8,4,5)$ & $(0.5,1,1.5)$ \\
$C_{6}$ & $(0.2,0.5,1)$ & $(2,2.5,3)$ & $(2.25,3,3.5)$ & $(0.5,1,2)$ \\
$C_{7}$ & $(5,7,9)$ & $(6,8,10)$ & $(2,3,5)$ & $(0,1,2)$ \\
\hline
\end{tabular}

Table 4. Normalized values of quantitative criteria

\begin{tabular}{ccc}
\hline$C$. & $A_{1}$ & $A_{2}$ \\
\hline$C_{4}$ & $(0.4000,0.5000,0.6500)$ & $(0.0600,0.0860,0.1000)$ \\
$C_{5}$ & $(0.1304,0.2174,0.4783)$ & $(0.5217,0.6522,1.0000)$ \\
$C_{6}$ & $(0.0000,0.0909,0.2424)$ & $(0.5455,0.6970,0.8485)$ \\
$C_{7}$ & $(0.1000,0.3000,0.5000)$ & $(0.0000,0.2000,0.4000)$ \\
\hline
\end{tabular}

Table 5. Normalized values of quantitative criteria (cont.)

\begin{tabular}{ccc}
\hline$C$. & $A_{3}$ & $A_{4}$ \\
\hline$C_{4}$ & $(0.6200,0.7740,1.0000)$ & $(0.0000,0.0500,0.0794)$ \\
$C_{5}$ & $(0.2000,0.3043,0.3913)$ & $(0.0000,0.0435,0.0870)$ \\
$C_{6}$ & $(0.6212,0.8485,1.0000)$ & $(0.0909,0.2424,0.5455)$ \\
$C_{7}$ & $(0.5000,0.7000,0.8000)$ & $(0.8000,0.9000,1.0000)$ \\
\hline
\end{tabular}

Table 6. Importance weights of criteria

\begin{tabular}{cccccc}
\hline$C$. & $D_{1}$ & $D_{2}$ & $D_{3}$ & $D_{4}$ & $D_{5}$ \\
\hline$C_{1}$ & VI & I & I & MI & I \\
$C_{2}$ & VI & MI & MI & VI & VI \\
$C_{3}$ & VI & MI & I & MI & VI \\
$C_{4}$ & LI & UI & LI & LI & UI \\
$C_{5}$ & VI & VI & I & VI & VI \\
$C_{6}$ & I & LI & UI & I & MI \\
$C_{7}$ & VI & VI & I & I & MI \\
\hline
\end{tabular}

Table 7. Averaged importance weights

\begin{tabular}{ll}
\hline$C$. & Averaged weights \\
\hline$C_{1}$ & $(0.40,0.65,0.85)$ \\
$C_{2}$ & $(0.65,0.90,1.00)$ \\
$C_{3}$ & $(0.55,0.80,0.95)$ \\
$C_{4}$ & $(0.00,0.15,0.40)$ \\
$C_{5}$ & $(0.65,0.90,0.95)$ \\
$C_{6}$ & $(0.20,0.40,0.65)$ \\
$C_{7}$ & $(0.50,0.75,0.90)$ \\
\hline
\end{tabular}

Table 8. Final fuzzy evaluation values

\begin{tabular}{ccccc}
\hline \multirow{2}{*}{ Values } & \multicolumn{4}{c}{ Alternatives } \\
\cline { 2 - 5 } & $A_{1}$ & $A_{2}$ & $A_{3}$ & $A_{4}$ \\
\hline$I_{i 1}$ & 0.0364 & 0.0374 & 0.0399 & 0.0277 \\
$I_{i 2}$ & 0.0289 & 0.0241 & 0.0291 & 0.0266 \\
$J_{i 1}$ & 0.1382 & 0.1561 & 0.1508 & 0.1 \\
$J_{i 2}$ & -0.2595 & -0.2562 & -0.2614 & -0.2029 \\
$Q_{i}$ & 0.1287 & 0.1645 & 0.1195 & 0.0940 \\
$Y_{i}$ & 0.3024 & 0.3597 & 0.3102 & 0.2217 \\
$Z_{i}$ & 0.5330 & 0.5911 & 0.5425 & 0.3980 \\
\hline
\end{tabular}

Table 9. Centroid points

\begin{tabular}{ccccr}
\hline \multirow{2}{*}{ Values } & \multicolumn{4}{c}{ Alternatives } \\
\cline { 2 - 5 } & $A_{1}$ & $A_{2}$ & $A_{3}$ & $A_{4}$ \\
\hline$x$ & 0.3010 & 0.3198 & 0.3083 & 0.2425 \\
$y$ & 0.3344 & 0.3351 & 0.3347 & 0.3335 \\
\hline
\end{tabular}

Table 10. Euclidean distance

\begin{tabular}{ccccr}
\hline \multirow{2}{*}{ Values } & \multicolumn{4}{c}{ Alternatives } \\
\cline { 2 - 5 } & $A_{1}$ & $A_{2}$ & $A_{3}$ & $A_{4}$ \\
\hline Distance & 0.4499 & 0.4632 & 0.4551 & 0.4124 \\
\hline
\end{tabular}

Based on Table 10, the alternative candidate $\left(A_{2}\right)$ has highest score; therefore, company should choose $A_{2}$ as its consulting firm. This numerical example has clearly demonstrated the computational procedure of the suggested model. A numerical comparison as well as sensitivity analysis may be conducted in the extension of this work in the future for further justification.

\section{Conclusions}

A centroid ranking approach based fuzzy MCDM method is proposed to evaluate and select consulting firms, where criteria are classified to qualitative, benefit quantitative and cost quantitative ones. In the proposed model, ratings of alternatives versus qualitative criteria and the importance weights of all criteria are assessed in linguistic values represented by fuzzy numbers. Membership functions of the final evaluation values can be developed. The Euclidean distance based on centroid points is applied to defuzzify all the final fuzzy evaluation values to determine the ranking order of alternatives in order to complete the model. Formulas for the two centroid points on horizontal and vertical axes can be clearly developed. Finally, a numerical example has demonstrated the computational process of the suggested model.

The suggested model can also be applied to solve many other fuzzy management problems in a multiple criteria environment. However, when applied, the following problems are worth closer examination:

(1) The outcome could be different if number of decision makers, linguistic ratings of various alternatives under different qualitative criteria, linguistic weightings for criteria, normalization formula, ... etc. are different.

(2) A case study is needed to better test the 
effectiveness of the suggested model. In addition, the number of criteria can be adjusted upon different cases if needed.

(3) In the suggested model, the criteria structure is limited to one level. This may not be enough. If some parent criteria contain several sub-criteria and some of these sub-criteria further include several sub-sub-criteria, a hierarchical structure must be needed to describe this situation.

\section{Acknowledgments}

The author would like to thank anonymous referees for their insights and suggestions, which make a better presentation of this work. Special thanks to Elianti and Nguyen Khoa $\mathrm{Tu}$ Uye for their data collecting and typing. This work was supported in part by National Science Council, Taiwan, R.O.C., under Grant NSC 98-2410-H-218-008-MY2.

\section{References}

[1] S. Abbasbandy and B. Asady, Ranking of fuzzy numbers by sign distance, Information Sciences, 176 (6), 2405-2416, 2006.

[2] S. Abbasbandy and T. Hajjari, A new approach for ranking of trapezoidal fuzzy numbers, Computers and Mathematics with Applications, 57, 413-419, 2009.

[3] W. Altman, What's the point of management consultants? Engineering \& Technology, 3 (12), 80-83, 2008.

[4] B. Asady, The revised method of ranking LR fuzzy number based on deviation degree, Expert Systems with Applications, 37, 5056-5060, 2010.

[5] G. Bortolan and R. Degani, A review of some methods for ranking fuzzy numbers, Fuzzy Sets and Systems, 15 (1), 1-19, 1985.

[6] C. Carlsson and R. Fullèr, Fuzzy multiple criteria decision making: Recent developments, Fuzzy Sets and Systems, 78 (2), 139-153, 1996.

[7] U. Cebeci and D. Ruan, A multi-attribute comparison of Turkish quality consultants by fuzzy AHP, International Journal of Information Technology \& Decision Making, 6 (1), 191-207, 2007.

[8] C.T. Chen, A fuzzy approach to select the location of the distribution center, Fuzzy Sets and Systems, 118 (1), 65-73, 2001

[9] S.J. Chen and C.L. Hwang, Fuzzy Multiple Attribute Decision Making, Springer, Berlin, 1992.

[10] C.C. Chou, A fuzzy MCDM method for solving marine transshipment container port selection problems, Applied Mathematics and Computation, 186 (1), 435-444, 2007.

[11] T.Y. Chou, S.T. Chou and G.H. Tzeng, Evaluating IT/IS investments: A fuzzy multi-criteria decision model approach, European Journal of Operational Research, 173 (3), 1026-1046, 2006.

[12] T.C. Chu and Y.C. Lin, An extension to fuzzy
MCDM, Computers \& Mathematics with Application, 27 (3), 445-454, 2009.

[13] F. Creplet, D. Dupouet, F. Kem, B. Mehmanpazir and F. Munier, Consultants and experts in management consulting firms, Research Policy, 30 (9), 1517-1535, 2001

[14] D. Dubois and H. Prade, Operations on fuzzy numbers, International Journal of Systems Science, 9 (6), 613-626, 1978.

[15] A. Kaufmann and M.M. Gupta, M.M., Introduction to Fuzzy Arithmetic: Theory and Application, Van Nostrand Reinhold, New York, 1991.

[16] X.W. Liu and S.L. Han, Ranking fuzzy numbers with preference weighting function expectations, Computers and Mathematics with Applications, 49 (11-12), 1731-1753, 2005.

[17] S. Önüt, S.S. Kara and E. Işik, Long term supplier selection using a combined fuzzy MCDM approach: A case study for a telecommunication company, Expert Systems with Applications, 36, 3887-3895, 2009.

[18] R.A. Ribeiro, Fuzzy multiple attribute decision making: A review and new preference elicitation techniques, Fuzzy Sets and Systems, 78 (2), 155-181, 1996.

[19] M. Saremi, S.F. Mousaui and A. Sanayei, TQM consultant selection in SMEs with TOPSIS under fuzzy environment, Expert Systems with Application, 36 (2), 2742-2749, 2009.

[20] E. Triantaphyllou and C.T. Lin, Development and evaluation of five fuzzy multi-attribute decision-making methods, International Journal of Approximate Reasoning, 14 (4), 281-310, 1996.

[21] T.G. Wang and H.F. Chen, Effects of internal support and consultant quality on the consulting process and ERP system quality, Decision Support Systems, 42 (2), 1029-1041, 2006.

[22] X. Wang and E.E. Kerre, Reasonable properties for the ordering of fuzzy quantities (I) \& (II), Fuzzy Sets and Systems, 118 (3), 375-385, 387-405, 200

[23] Y.J. Wang and H.S. Lee, The revised method of ranking fuzzy numbers with an area between the centroid and original points, Computers \& Mathematics with Applications, 55 (9), 2033-2042, 2008.

[24] Y.M. Wang, J.B. Yang, D.L. Xu and K.S. Chin, 2006. On the centroids of fuzzy numbers, Fuzzy Sets and Systems, 157 (7), 919 - 926, 2006.

[25] D. Yong, Z. Zhu and Q. Liu, Q., Ranking fuzzy numbers with an area method using radius of gyration, Computers and Mathematics with Applications, 51 (6-7), 1127-1136, 2006.

[26] L.A. Zadeh, The concept of a linguistic variable and its application to approximate reasoning, part 1, 2 and 3, Information Sciences, 8 (3/4), 199-249/301-357, 1975; 9 (1), 43-80, 1976.

[27] L.A. Zadeh, L.A., Fuzzy set, Information and control, 8 (3), 338-353, 1965. 